\title{
Prevalence, Risk Factors And Location Of Skip Metastasis In Papillary Thyroid Carcinoma: A Systematic Review And Meta-Analysis
}

This article was published in the following Dove Press journal: Cancer Management and Research

\author{
Yuxuan Qiu (iD) ${ }^{1, *}$ \\ Yuan Fei ${ }^{1} *$ \\ Jingyan $\mathrm{Liu}^{2}$ \\ Chang $\mathrm{Liu}^{3}$ \\ Xin $\mathrm{He}^{4}$ \\ Ning Zhu ${ }^{5}$ \\ Wan-jun Zhao' \\ Jing-qiang Zhu' \\ 'Department of Thyroid \& Parathyroid \\ Surgery, West China Hospital, Sichuan \\ University, Chengdu, People's Republic of \\ China; ${ }^{2}$ Department of Ultrasound, West \\ China Hospital, Sichuan University, \\ Chengdu, People's Republic of China; \\ ${ }^{3}$ Public Policy and Administration, \\ Department of Government, London \\ School of Economics and Political \\ Science, London, UK; ${ }^{4}$ West China \\ School of Medicine, West China Hospital, \\ Sichuan University, Chengdu, People's \\ Republic of China; ${ }^{5}$ Library and \\ Information Science, School of \\ Information Management, Nanjing \\ University, Nanjing, People's Republic of \\ China
}

*These authors contributed equally to this work
Correspondence: Jing-qiang Zhu Department of Thyroid \& Parathyroid surgery, West China Hospital, Sichuan University, No. 37 Guo Xue Xiang, Chengdu, Sichuan 61004I, People's Republic of China

$\mathrm{Tel} / \mathrm{Fax}+86-28-85422467$

Email zjq-wkys@163.com
Background: Skip metastasis is a special type in cervical lymph node metastasis (LNM) of patients diagnosed with papillary thyroid carcinoma (PTC) which induced poor prognosis. There are few studies about skip metastasis and conclusions remained uncertain. Therefore, this study aims to explore the frequency and to investigate risk factors of skip metastasis in PTC.

Methods: Through searching the keyword by PubMed and Embase databases which articles published up to $1^{\text {st }}$ August 2018 about skip metastasis in papillary thyroid carcinoma, we extract data in order to assure whether those materials meet the criteria.

Results: The prevalence of skip metastasis is $12.02 \%$ in light of our meta-analysis of 18 studies with 2165 patients. The upper pole location $(\mathrm{RR}=3.35,95 \% \mathrm{CI}=1.65-6.79, \mathrm{P}=0.0008)$ and tumors size $\leq 1 \mathrm{~cm}(\mathrm{RR}=2.65,95 \% \mathrm{CI}=1.50-4.70, \mathrm{P}=0.0008)$ are significantly associated with skip metastasis, whereas lymphovascular invasion $(\mathrm{RR}=0.33,95 \% \mathrm{CI}=0.15-0.75, \mathrm{P}=0.0083)$ exists lower rate of skip metastasis. Multifocality, gender, age, bilaterality, thyroiditis and Extrathyroidal extension (ETE) are insignificantly associated with skip metastasis. Level II and level III are the most frequently affected areas.

Conclusion: The lateral compartment should be carefully examined especially for those PTC patients who present primary tumors in the upper lobe with a primary tumor size $\leq 10$ mm which could be detected with skip metastasis.

Keywords: papillary thyroid carcinoma, skip metastasis, lateral neck dissection, metaanalysis

\section{Introduction}

Over the past three decades, the incidence of papillary thyroid cancer (PTC) has increased threefold, where the PTC accounts for approximately $90 \%$ of thyroid cancer. $^{1-3}$ Nevertheless, prognosis of PTC is relatively good and the mortality is stable at the rate of approximately 0.5 deaths per $100,000 .^{2}$ Thus, up to $95 \%$ of patients with PTC can be cured by surgery and accessory therapies. ${ }^{3}$ PTC tends to metastasize to cervical lymph nodes the first time. Cervical lymph nodes are divided into two main areas. Central lymph nodes (CLN) are called as level VI, including precricoid (Delphian), pretracheal, paratracheal, and perithyroidal nodes. ${ }^{4}$ Lateral lymph nodes (LLN) include levels II, III, IV, and V, of which Level II extends from skull-based to the level of inferior border of hyoid bone for which medial boundary is the stylohyoid muscle and lateral boundary is posterior border of sternocleidomastoid (SCM) muscle. The nodes of Level III extend from the 
inferior border of the hyoid bone to the inferior border of the cricoid cartilage, level IV nodes extend from the inferior border of the cricoid cartilage to the clavicle. Medial and lateral boundaries of these two levels are the same as Level II. Level $\mathrm{V}$ is also called the posterior triangle group, including the superior boundary which is the apex formed by convergence of the SCM and trapezius muscle, the inferior boundary which is clavicle, the medial boundary which is the posterior border of the SCM muscle, and the lateral boundary which is the anterior border of the trapezius muscle. ${ }^{5}$ Generally, ipsilateral CLN is the first step of lymph nodes metastasis, and then it metastasizes to contralateral CLN and finally to LLN. According to different studies on postoperative histology of metastatic lymph nodes, the rate of CLNM is $14.6-52 \%$, and the rate of both CLNM and LLNM are $42-65 \%$. $^{6,7}$ The rate of only LLNM with negative CLNM defined as skip metastasis is about $3-19.7 \% .^{8-11}$

To treat lymph node metastasis, prophylactic CLN dissection (CLND) is accepted as a standard treatment in the world, while whether LLN dissection (LLND) can be prevented is still under discussion. ${ }^{4}$ Various thyroid centers reached a consensus that it was an overtreatment of clearing LLN without histologic basis. Meanwhile, larger operative extent would prolong the surgery and give rise to additional complications including intractable chyle leakage and injuries of vagus nerve, accessory nerve, hypoglossal nerve, or facial nerve. ${ }^{12}$ However, in case of locoregional recurrence, cleaning tumor foci is of crucial importance during the first operation. Otherwise, patients will be confronted with reoperation and greater risks. ${ }^{13} \mathrm{At}$ present, according to the 2015 ATA guidelines, most thyroid surgeons make therapeutic dissection of LLN under the evidence of histologic metastatic tumor thus clearing tumor foci and reducing complications. ${ }^{14}$ Therefore, to identify the involved lateral lymph nodes accurately before the surgery is urgent.

Metastatic lymph nodes are commonly under ultrasound examination, followed by CT and MRI. Sonographic features of metastatic lymph nodes include enlargement, calcifications, loss of the fatty hilum, hyperechogenicity, a rounded rather than oval shape, cystic change, and peripheral vascularity. ${ }^{14}$ Sometimes, abnormal lymph nodes may not be found when it was microcalcification or overlaid by thyroid tissue. ${ }^{15}$ On the basis of present reports, the preoperative sonographic sensitivity in initial patients is about $30-55 \%$ for central compartment, and $62-94 \%$ for lateral compartment. ${ }^{16,17} \mathrm{CT}$ or
MRI can be used for patients' suspected clinically apparent multiple or bulky lymph node involvement, and preoperative 18FDG-PET scanning is not recommended to be used frequently. According to various studies, the false-positive and false-negative rate of ultrasound to palpable LLN both range from $20 \%$ to $30 \% .{ }^{18}$ In addition, in previous studies, ultrasonic examination cannot identify abnormal lymph node for which the diameter is less than $5 \mathrm{~mm} .{ }^{19}$

Hence, determining the risk factors of skip metastasis can guide performers strengthen the awareness when CLN is negative and lead them to choose appropriate extent of procedure. Features of normal LLNM were discussed by So et al, but the conclusions of skip metastasis were still controversial. $^{20}$ There are few studies focusing on predictive factors of skip metastasis. Therefore, the aim of this meta-analysis is to explore the frequency and investigate risk factors of skip metastasis in PTC.

\section{Methods}

Our meta-analysis was registered to PROSPERO (https:// www.crd.york.ac.uk/PROSPERO) and ID was CRD4201808 7681.

\section{Search Strategy}

We searched PubMed and Embase databases up to 1st August 2018. The search terms were the following: 1) papillary thyroid cancer OR papillary thyroid carcinoma OR papillary thyroid neoplasm OR PTC; 2) skip metastasis OR skip metastases OR lateral lymph node OR lateral compartment. Two independent investigators do the study selection by screening the titles and abstracts; meanwhile, relevant reviews and reference lists were also checked. Discrepancies were resolved by discussion.

\section{Selection Criteria}

We included studies which met the following criteria:

1. Prospective or retrospective cohort studies;

2. PTC and LNM were confirmed by histopathology;

3. Definition of skip metastasis corresponded to our article;

4. Patients were under thyroidectomy and CLND or LLND;

5. Any one of the prevalence or risk factors or location of skip metastasis were available;

6. English studies.

We excluded studies by the following criteria: 
1. Reviews, case reports, letters to the editor, conference abstracts, and so on;

2. Studies enrolled patients with follicular thyroid carcinoma, medullary thyroid carcinoma, or anaplastic carcinoma;

3. Sample size less than 10 .

\section{Data Extraction And Quality Assessment}

Each eligible study was extracted by two independent reviewers. Study characteristics were needed including first author, publication years, countries of study, study design, study population (PTC or PTMC), number of patients, surgical extent, indication of LLND (prophylactic or therapeutic). Outcomes including prevalence, risk factors, and location of skip metastasis were extracted. The risk factors included multifocality, sex, tumor location, age, tumor size, lymphovascular invasion, tumor bilaterality, thyroiditis, extrathyroidal extension (ETE), and capsule invasion.

We assessed the quality of each eligible study using Newcastle-Ottawa Scale (NOS), ${ }^{21}$ which offers capacity in evaluating cohort studies by a total of eight items of three major parts, including the study population selection (selection), comparability (comparability), and result (outcome). NOS uses the semi-quantitative principles of star system to do quality assessment and full score is 9 stars. All quality assessments were performed by two independent reviewers.

\section{Statistical Analysis}

Statistical analysis was performed using the $\mathrm{R}$ version 3.5.1 (Vienna, Austria; https://www.r-project.org) plus RStudio version 1.1.456 (250 Northern Ave, Boston, MA 02210) with "meta" package (version 4.9-2). For prevalence and location of skip metastasis, ratios were calculated using Freeman-Tukey double arcsine transformation..$^{22}$ For each risk factor, the relative risk ratio (RR) was calculated with a $95 \%$ confidence interval (CI). A P-value $<0.05$ was considered statistically different. Heterogeneity was examined by using Q-test and I2 statistic. Random effects model was used when studies were heterogeneous $\left(\mathrm{P}<0.1\right.$ or $\mathrm{I}^{2}>50 \%$ ); otherwise, fixed-effects model was applied. ${ }^{23}$ There are still some biases about Egger's linear regression test and Begg's rank correlation test or funnel plot.

\section{Results}

After searching the databases, 646 studies from PubMed and 967 studies from Embase were initially found and 1047 articles were non-overlapping articles. A total of 169 articles were excluded because they were not written in English and 394 studies were also excluded due to the fact that they are reviews, letters, case reports, and conference abstracts. After scanning the titles and abstracts of the remaining 484 studies, 31 articles were subjected to a full-text evaluation. After the full-text review, 20 studies met our selection criteria. However, four of those twenty studies were excluded because the study population is overlapping. Finally, a total of 18 studies were included in our metaanalysis. All the patients included in those studies were with LLNM who may not be with CLNM and were pN1b patients. Patients in those studies were first diagnosed or highly suspicious of LNM by ultrasound, and then they under went fine-needle aspiration (FNA) biopsy to check LNM. Finally, a thyroidectomy with CLND which has a modified or selective LLND (therapeutic) was performed. If FNA biopsy could not determine the nature of lymph nodes, intra-operational frozen biopsy was applied. Thus, all patients with skip metastasis were confirmed by histopathology. Flow chart with details was displayed in Figure 1 and for study characteristics, see Table1.

\section{Prevalence Of Skip Metastasis}

All 18 studies reported the prevalence of skip metastasis in each LLNM population. ${ }^{8-11,24-37}$ Overall, 260 patients $(12.02 \%)$ had to skip metastasis in the sample of 2165 patients who had LLNM. The ratio of skip metastasis ranged from $5 \%$ to $25 \%$ (median $13.16 \%$, inter-quartile range 8.22 $19.56 \%)$. A random-effects model was used because of the relatively stable heterogenicity $\left(\mathrm{P}<0.0001, \mathrm{I}^{2}=65.3 \%\right)$. The prevalence of skip metastasis was $12.02 \%(95 \% \mathrm{CI}=9.45 \%$ $14.82 \%$ ). No significant publication bias was found in Begg's rank correlation test $(\mathrm{P}=0.3065)$ and Egger's linear regression test $(\mathrm{P}=0.1730$; Figure 2$)$.

\section{Risk Factors For Skip Metastasis}

All risk factors for skip metastasis are shown in Table 2.

\section{Multifocality Of Tumor}

Data of 7 included studies were analyzed using fixed effects model $\left(\mathrm{P}=0.0849, \mathrm{I}^{2}=46.0 \%\right) \cdot{ }^{8-11,24-26}$ Tumor multifocality was not significantly associated with skip metastasis $(\mathrm{RR}=0.95,95 \% \mathrm{CI}=0.65-1.40, \mathrm{P}=0.8047)$.

\section{Gender (Male)}

A fixed effects model was applied owing to little heterogenicity in 6 enrolled articles $\left(\mathrm{P}=0.4578, \mathrm{I}^{2}=0.0 \%\right) .{ }^{9-11,24-26}$ 


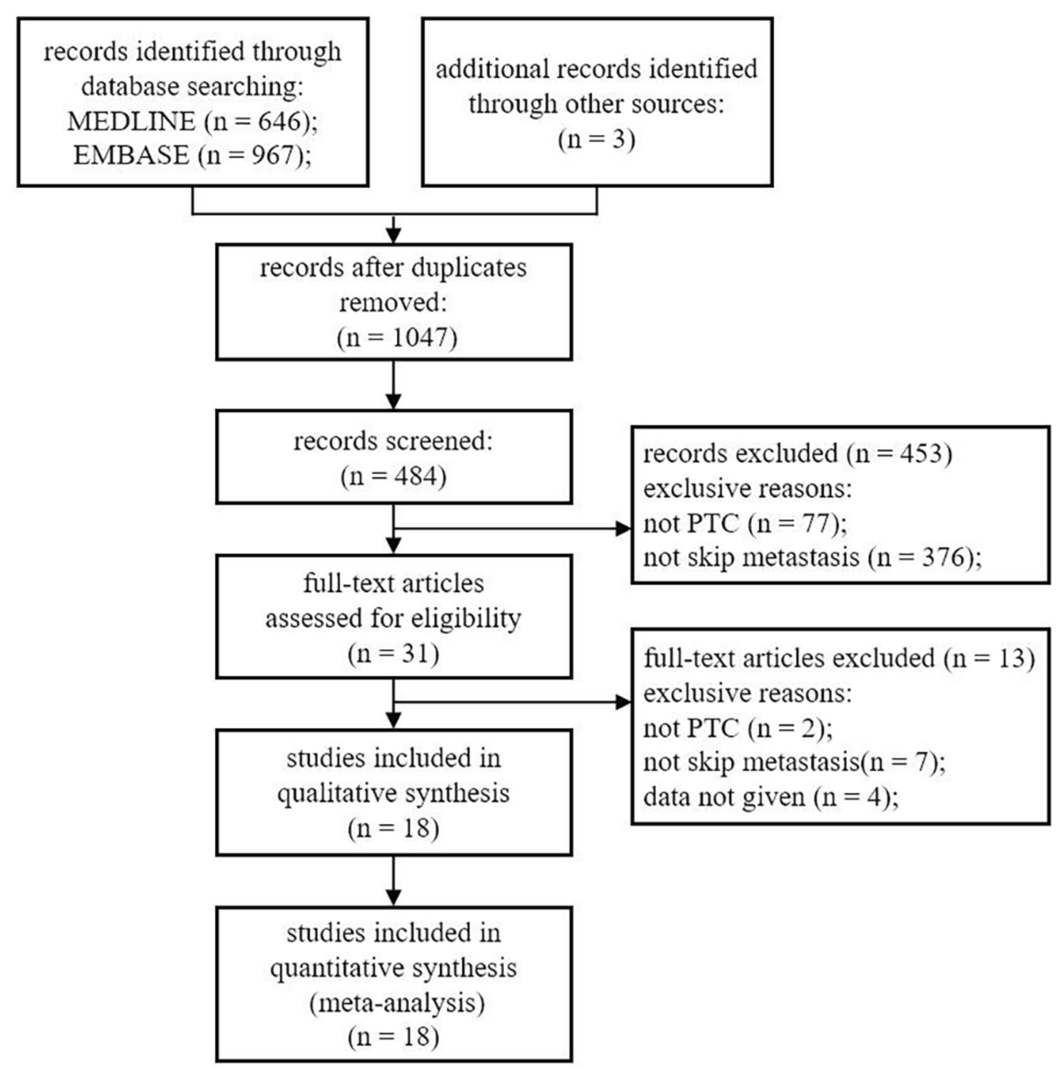

Figure I PRISMA flow chart of the included studies.

Gender was not statistically related to skip metastasis $(\mathrm{RR}=0.94,95 \% \mathrm{CI}=0.68-1.30, \mathrm{P}=0.4578)$.

\section{Tumor Location}

Six studies reported the association between tumor location (upper pole) and skip metastasis. ${ }^{9-11,24-26}$ Data were analyzed by random effects model $\left(\mathrm{P}=0.0019, \mathrm{I}^{2}=73.7 \%\right)$. Tumors located in the upper pole had a significantly higher ratio for skip metastasis than those located in the mid-lower pole $(\mathrm{RR}=3.35,95 \% \mathrm{CI}=1.65-6.79, \mathrm{P}=0.0008$; Figure 3$)$.

\section{Age ( $<45$ Years)}

A fixed effects model was used $\left(\mathrm{P}=0.3293, \mathrm{I}^{2}=13.3 \%\right)$ to analyze data from 5 studies..$^{9,11,24-26}$ Age ( $<45$ years) was not significantly connected with skip metastasis $(\mathrm{RR}=$ $0.84,95 \% \mathrm{CI}=0.63-1.14, \mathrm{P}=0.2685$ ).

\section{Tumor Size $(\leq \mathrm{I} \mathrm{Cm})$}

A medium to high heterogenicity was detected ( $\mathrm{P}=$ $\left.0.0027, \mathrm{I}^{2}=72.5 \%\right)$ in 6 articles and a random effects model was applied. ${ }^{9-11,24-26}$ Tumors $(\leq 1 \mathrm{~cm})$ existed a significantly higher odd for skip metastasis than those $>1$ $\mathrm{cm}(\mathrm{RR}=2.65,95 \%$ CI $=1.50-4.70, \mathrm{P}=0.0008$; Figure 4).

\section{Lymphovascular Invasion}

Only two articles reported outcomes of lymphovascular invasion and a fixed effects model was applied $(\mathrm{P}=0.9872$, $\left.\mathrm{I}^{2}=0.0 \%\right) .{ }^{9,10}$ Lymphovascular invasion has a statistically lower rate of skip metastasis $(\mathrm{RR}=0.33,95 \% \mathrm{CI}=0.15-0.75$, $\mathrm{P}=0.0083$; Figure 5).

\section{Tumor Bilaterality}

No obvious heterogenicity was found in 5 enrolled studies $\left(\mathrm{P}=0.7504, \mathrm{I}^{2}=0.0 \%\right)$. Data were analyzed by fixed effects model. ${ }^{8,10,11,24,26}$ Tumor bilaterality was not significantly associated with skip metastasis $(\mathrm{RR}=1.08,95 \%$ $\mathrm{CI}=0.74-1.56, \mathrm{P}=0.6989$ ).

\section{Thyroiditis}

A fixed effects model was used in 2 studies $(\mathrm{P}=0.2048$, $\left.\mathrm{I}^{2}=37.8 \%\right) .{ }^{24,26}$ No significant difference was found in PTC patients with or without thyroiditis $(\mathrm{RR}=1.36,95 \%$ $\mathrm{CI}=0.88-2.10, \mathrm{P}=0.1646$ ).

\section{Extrathyroidal Extension (ETE)}

Six studies had outcomes of ETE and a fixed effects model was applied $\left(\mathrm{P}=0.2488, \mathrm{I}^{2}=24.7 \%\right) .{ }^{8-10,24-26}$ ETE was 


\begin{tabular}{|c|c|c|c|c|c|c|}
\hline 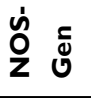 & $\infty \wedge \infty a$ & 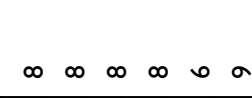 & $\infty \quad \infty \quad \infty \wedge$ & $r$ & $\infty$ & $r$ \\
\hline 号蓠 & \multicolumn{6}{|c|}{ 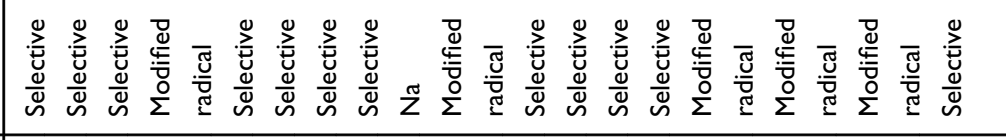 } \\
\hline 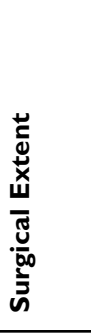 & 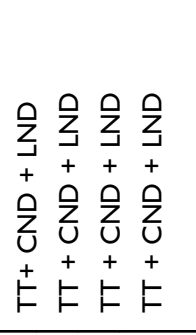 & 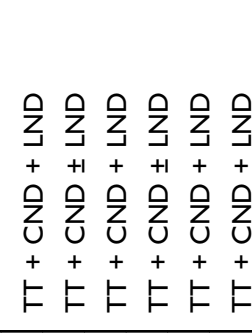 & 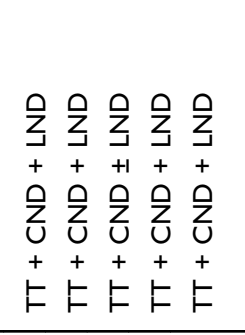 & 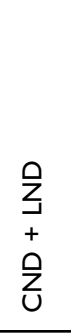 & 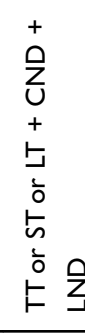 & 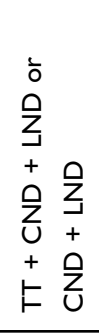 \\
\hline $\begin{array}{l}\frac{0}{\tilde{๊}} \\
\text { है } \\
\text { एँ }\end{array}$ & 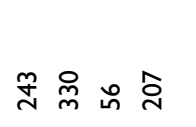 & 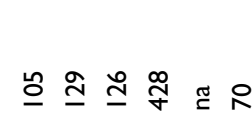 & 。ํํํ $\overline{\bar{N}}$ 요 & $\leq$ & $\underline{\underline{n}}$ & $\stackrel{\pi}{\Sigma}$ \\
\hline$\frac{\frac{0}{\pi}}{\Sigma}$ & 포 ‡ & స & 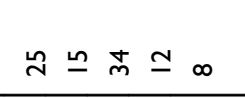 & n & $\stackrel{\infty}{\leftrightarrow}$ & $\Xi$ \\
\hline$\stackrel{8}{⿺}$ & 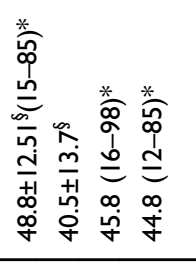 & 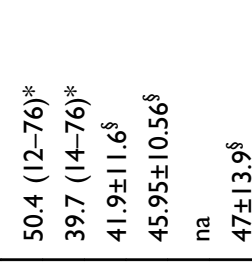 & 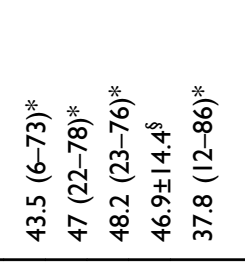 & 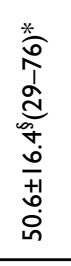 & 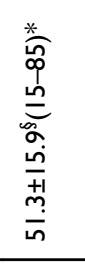 & $\stackrel{\pi}{\simeq}$ \\
\hline ט̃ & 耑品 \& & $\bar{m} \underline{\underline{y}} \underset{\dot{q}}{\circ}$ 子 & 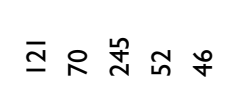 & $\pi$ & $\overline{\mathfrak{N}}$ & $\stackrel{\circ}{\circ}$ \\
\hline 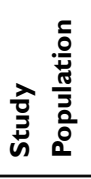 & 吕吕吕吕 & 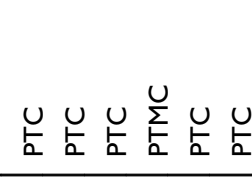 & 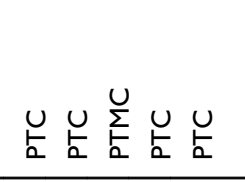 & 㫐 & U & $\underset{\substack{U \\
ٍ}}{\bigcup}$ \\
\hline 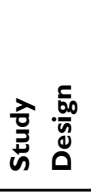 & 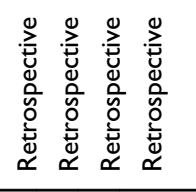 & 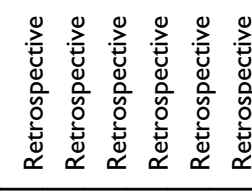 & 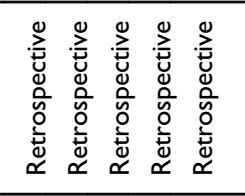 & 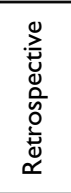 & 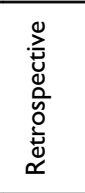 & 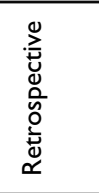 \\
\hline 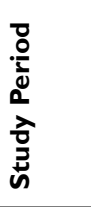 & 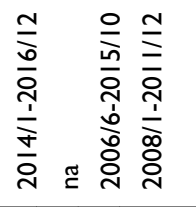 & 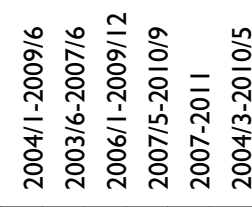 & 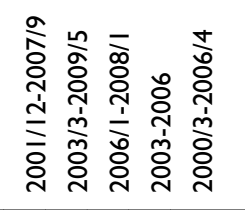 & $\begin{array}{l}\text { ¿े } \\
\text { ì } \\
\text { రัن }\end{array}$ & 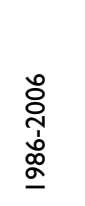 & 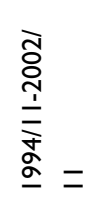 \\
\hline نे & 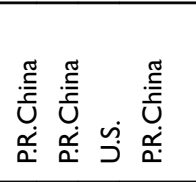 & 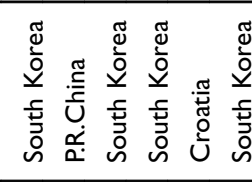 & 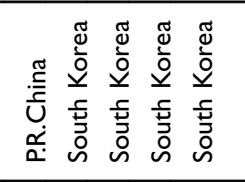 & 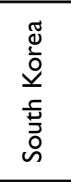 & 荵 & 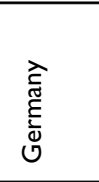 \\
\hline ঠ્ & 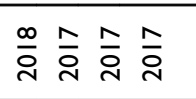 & 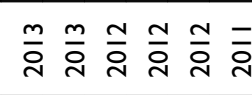 & 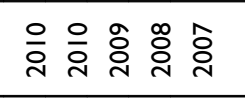 & ڤેণ & ڤे & ষ্ণ \\
\hline 产 & 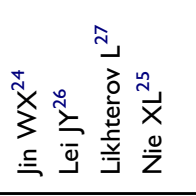 & 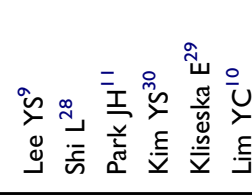 & 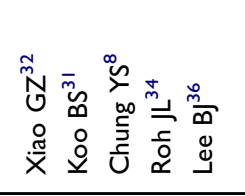 & 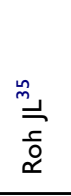 & 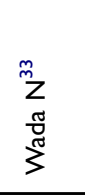 & 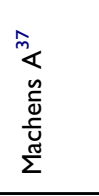 \\
\hline
\end{tabular}




Study
Jin WX 2018
Lei JY 2017
Likhterov L 2017
Nie XL 2017
Lee YS 2013
Shi L 2013
Park JH 2012
Kim YS 2012
Kliseska E 2012
Lim YC 2011
Xiao GZ 2010
Koo BS 2010
Chung YS 2009
Roh JL 2008
Lee BJ 2007
Roh JL 2007
Wada N 2007
Machens A 2004
Random effects model
Heterogeneity:

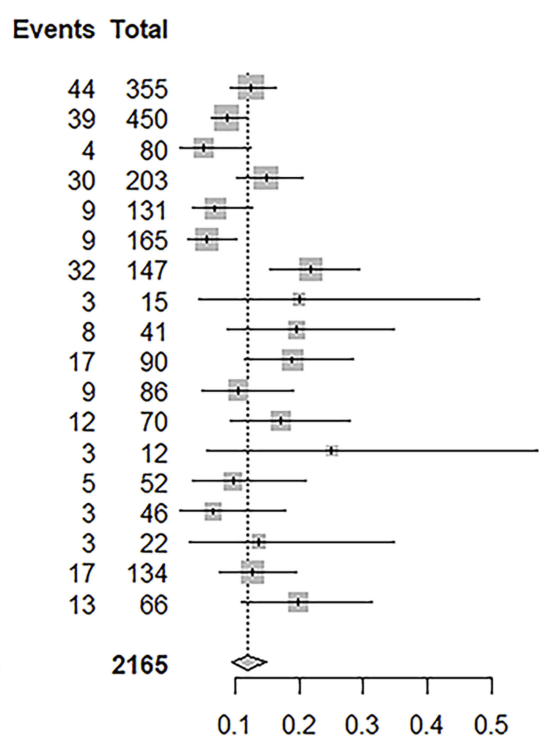

$\begin{array}{rrr}\text { Proportion } & 95 \%-C l & \text { Weight } \\ 0.1239 & {[0.0915 ; 0.1628]} & 8.5 \% \\ 0.0867 & {[0.0624 ; 0.1166]} & 8.7 \% \\ 0.0500 & {[0.0138 ; 0.1231]} & 5.7 \% \\ 0.1478[0.1020 ; 0.2042] & 7.6 \% \\ 0.0687[0.0319 ; 0.1264] & 6.8 \% \\ 0.0545[0.0252 ; 0.1010] & 7.3 \% \\ 0.2177[0.1539 ; 0.2932] & 7.0 \% \\ 0.2000[0.0433 ; 0.4809] & 2.0 \% \\ 0.1951[0.0882 ; 0.3487] & 4.0 \% \\ 0.1889[0.1141 ; 0.2851] & 5.9 \% \\ 0.1047[0.0490 ; 0.1894] & 5.8 \% \\ 0.1714[0.0918 ; 0.2803] & 5.3 \% \\ 0.2500[0.0549 ; 0.5719] & 1.7 \% \\ 0.0962[0.0320 ; 0.2103] & 4.6 \% \\ 0.0652 & {[0.0137 ; 0.1790]} & 4.3 \% \\ 0.1364 & {[0.0291 ; 0.3491]} & 2.7 \% \\ 0.1269 & {[0.0757 ; 0.1953]} & 6.8 \% \\ 0.1970[0.1093 ; 0.3132] & 5.2 \%\end{array}$

$0.1202[0.0945 ; 0.1482] 100.0 \%$ $I^{2}=65 \%, \tau^{2}=0.0041, p<0.01$

Figure 2 Prevalence of skip metastasis in patients with lateral lymph node metastasis.

Table 2 Risk Factors For Skip Metastasis

\begin{tabular}{|c|c|c|c|c|c|}
\hline & $\mathbf{R R}$ & $95 \% \mathrm{Cl}$ & $\mathbf{P}$ & $P$ value Of $Q$-test & $1^{2}$ \\
\hline Multifocality of tumor & 0.95 & $0.65-1.40$ & 0.8047 & 0.0849 & $46.0 \%$ \\
\hline Gender (male) & 0.94 & $0.68-1.30$ & 0.4578 & 0.4578 & $0.0 \%$ \\
\hline Tumor location (upper pole) & 3.35 & $1.65-6.79$ & 0.0008 & 0.0019 & $73.7 \%$ \\
\hline Age ( $<45$ years) & 0.84 & $0.63-1.14$ & 0.2685 & 0.3293 & $13.3 \%$ \\
\hline Tumor size $(\leq \mathrm{I} \mathrm{cm})$ & 2.65 & $1.50-4.70$ & 0.0008 & 0.0027 & $72.5 \%$ \\
\hline Lymphovascular invasion & 0.33 & $0.15-0.75$ & 0.0083 & 0.9872 & $0.0 \%$ \\
\hline Tumor bilaterality & 1.08 & $0.74-1.56$ & 0.6989 & 0.7504 & $0.0 \%$ \\
\hline Thyroiditis & 1.36 & $0.88-2.10$ & 0.1646 & 0.2048 & $37.8 \%$ \\
\hline Extrathyroidal extension (ETE) & 1.20 & $0.88-1.65$ & 0.2469 & 0.2488 & $24.7 \%$ \\
\hline Capsule invasion & 1.02 & $0.32-3.28$ & 0.9734 & 0.0004 & $83.5 \%$ \\
\hline
\end{tabular}

Abbreviations: $\mathrm{RR}$, risk ratio; $95 \% \mathrm{Cl}, 95 \%$ confidence interval.

not significantly associated with skip metastasis $(\mathrm{RR}=1.20,95 \% \mathrm{CI}=0.88-1.65, \mathrm{P}=0.2469)$.

\section{Capsule Invasion}

A high heterogenicity was found in 4 included studies $\left(\mathrm{P}=0.0004, \mathrm{I}^{2}=83.5 \%\right)$ and a random effects model was applied. ${ }^{8,10,24,26}$ Capsule invasion was not associated with skip metastasis $(\mathrm{RR}=1.02,95 \% \mathrm{CI}=0.32-3.28, \mathrm{P}=0.9734)$.

\section{Location Of Skip Metastasis}

Four studies reported outcomes of the location of skip metastasis and a total of 132 patients had skip metastasis. ${ }^{10,11,24,26}$ Seventy-six patients were skip metastasis positive in level II, 86 in level III, 18 in level IV, and 6 in level V. Ratio of skip metastasis was $56.29 \%(95 \% \mathrm{CI}=39.77-72.16 \%)$ in level II, $68.85 \%$ (95\% CI $=42.11-90.46 \%)$ in level III, $15.95 \%(95 \%$
$\mathrm{CI}=0.66-45.34 \%)$ in level $\mathrm{IV}$, and $3.67 \%(95 \% \mathrm{CI}=0.72-$ $8.11 \%$ ) in level V (Table 3 ).

\section{Subgroup Analysis}

Owing to the fact that 2 studies reported outcomes of PTMC, subgroup analysis was conducted in studies of PTC. ${ }^{8,30}$

Prevalence of skip metastasis in PTC was $11.88 \%$ $(95 \%$ CI $=9.33-14.68 \%)$ and heterogenicity remained stable $\left(\mathrm{P}<0.0001, \mathrm{I}^{2}=67.3 \%\right)$.

Tumor multifocality was not significantly associated with skip metastasis $(\mathrm{RR}=0.95,95 \% \mathrm{CI}=0.50-1.81, \mathrm{P}$ $=0.8838)$. However, heterogenicity increased $(\mathrm{P}=0.0533$, $\mathrm{I}^{2}=53.7 \%$ ) and random effects model was applied. Tumor bilaterality was not significantly associated with skip metastasis $(\mathrm{RR}=1.09,95 \% \mathrm{CI}=0.75-1.59, \mathrm{P}=0.6346)$ 


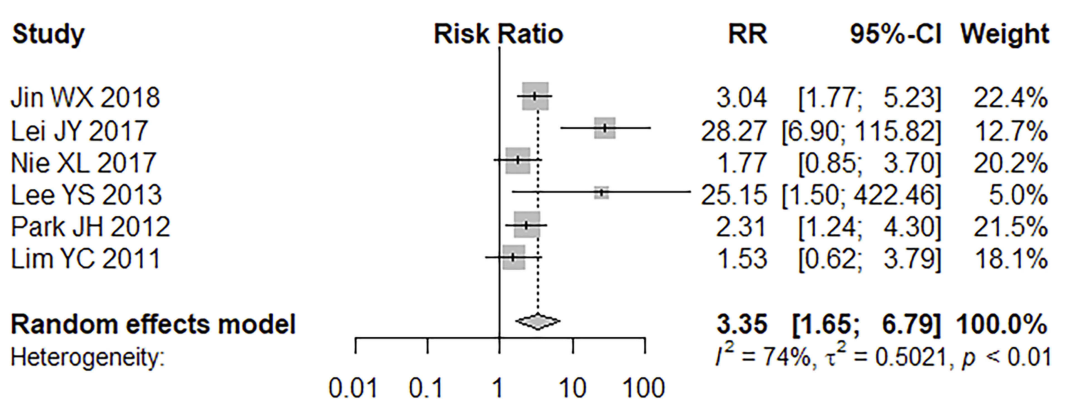

Figure 3 Forest plot of tumor location (upper pole).

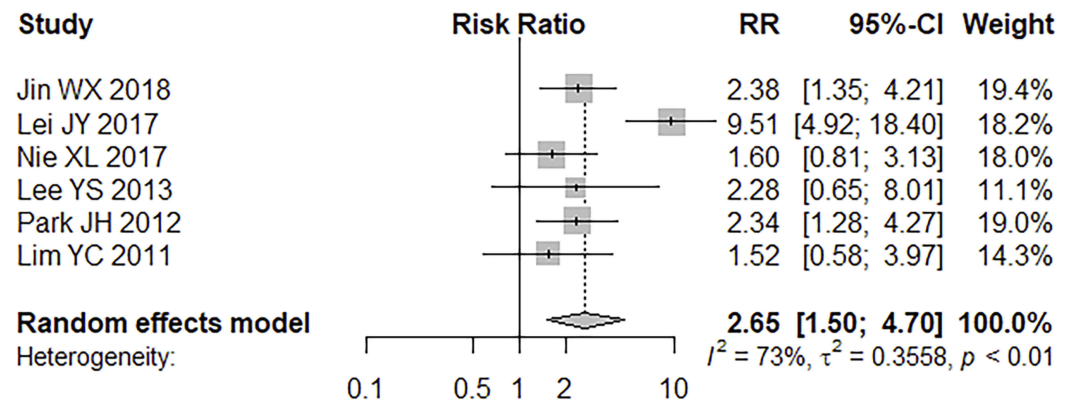

Figure 4 Forest plot of tumor size $(\leq \mathrm{lm})$.

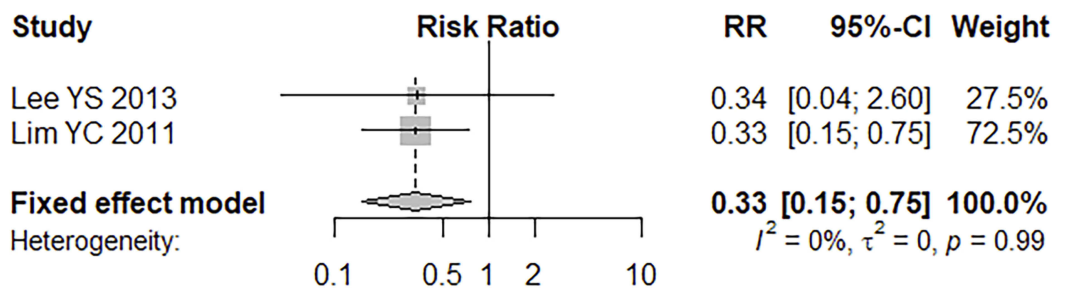

Figure 5 Forest plot of lymphovascular invasion.

Table 3 Location Of Skip Metastasis

\begin{tabular}{|l|l|l|l|l|l|}
\hline Level & No. Of Studies & Proportion \% & $\mathbf{9 5 \%} \mathbf{C l}$ & P value Of Q-test $^{\mathbf{I}^{2}}$ \\
\hline II & 4 & 56.29 & $39.77-72.16$ & 0.0162 & $70.9 \%$ \\
III & 4 & 68.85 & $42.11-90.46$ & $<0.001$ & $89.2 \%$ \\
IV & 4 & 15.95 & $0.66-42.34$ & $<0.00 I$ & $90.8 \%$ \\
V & 4 & 3.67 & $0.72-8.11$ & 0.2348 & $29.6 \%$ \\
\hline
\end{tabular}

and heterogeneity did not change obviously $\left(\mathrm{P}=0.6362, \mathrm{I}^{2}\right.$ $=0.0 \%$ ). Extrathyroidal extension did not exhibit a significant association with skip metastasis $(\mathrm{RR}=1.21,95 \%$ $\mathrm{CI}=0.88-1.66, \mathrm{P}=0.2422)$ but heterogenicity slightly increased $\left(\mathrm{P}=0.1580, \mathrm{I}^{2}=39.5 \%\right)$. Capsule invasion was not significantly associated $(\mathrm{RR}=1.25,95 \% \mathrm{CI}=0.33$ 4.69, $\mathrm{P}=0.7460)$ and heterogenicity was still high $(\mathrm{P}=$ $\left.0.0003, \mathrm{I}^{2}=87.9 \%\right)$.

\section{Sensitive Analysis}

Sensitive analysis was conducted in factors where heterogenicity existed.

Heterogenicity of prevalence of skip metastasis did not decrease significantly when omitting any article. However, heterogenicity of tumor size significantly decreased $\left(\mathrm{I}^{2}=0.0 \%\right)$ and tumors $(\leq 1 \mathrm{~cm})$ existed a surprisingly higher odd for skip metastasis than those $>1 \mathrm{~cm}(\mathrm{RR}=2.08,95 \%$ 
$\mathrm{CI}=1.51-2.86, \mathrm{P}<0.0001)$, when omitting one study. ${ }^{26}$ Capsule invasion was not greatly associated with skip metastasis $(\mathrm{RR}=0.64,95 \% \mathrm{CI}=0.30-1.36, \mathrm{P}<0.2437)$ after omitting one study $\left(\mathrm{I}^{2}=42.4 \%\right){ }^{26}$ About location of skip metastasis, ratio was $65.19 \%(95 \% \mathrm{CI}=55.34-74.46 \%)$ in level II and 55.03\% (95\% CI $=44.98-64.89 \%)$ in level III and heterogenicity decreased remarkably $\left(\mathrm{I}^{2}=0.0 \%\right)$ when omitting this study. ${ }^{11}$ Ratio was $5.17 \%(95 \% \mathrm{CI}=1.51-$ $10.38 \%)$ in level IV and $3.67 \%(95 \% \mathrm{CI}=0.72-8.11 \%)$ in level $\mathrm{V}$ in both levels $\left(\mathrm{I}^{2}=0.0 \%\right)$ when omitting this article. $^{10}$

\section{Discussion}

Lymph node metastasis has been reported in 30-80\% PTC patients including PTMC. ${ }^{38}$ It is controversial whether LNM is associated with a relatively poor prognosis. However, there is a consensus that LNM will increase the risk of lymph node recurrence and the reoperation of recurrence will increase operative complications and also increase medical costs to some degree. ${ }^{39-41}$

In our meta-analysis, the prevalence of skip metastasis was $12.02 \%(95 \% \mathrm{CI}=9.45-14.82 \%)$, and ranged from $5 \%$ to $25 \%$, which could be explained by different sample sizes and regions. Most of the studies (11/18) had limited number of patients $(<100)$; as a result, that the incidence of skip metastasis was very low, ${ }^{20}$ especially when the rate of normal LNM was $20.9 \%$. Therefore, as a rare event in PTC patients, it might be ignored by clinicians who advocated routine CLND given that the central compartment is the first site of lymph node metastasis. Then, LNM involves ipsilateral lateral compartment and followed by the contralateral lateral compartment and the mediastinal lymph nodes. ${ }^{42,43}$ This regularity might also contribute to the ignorance of skip metastasis.

In our meta-analysis, tumors located in upper lode, no larger than $1 \mathrm{~cm}$, and without lymphovascular invasion, were more likely to have skip metastasis, while multifocality, gender, age, bilaterality, thyroiditis, and ETE were not significantly associated with skip metastasis.

Tumors presented in the upper pole of thyroid were significant risk factors of skip metastasis. A tumor located in the upper pole thyroid may migrate along the superior thyroid artery to lateral lymph nodes and bypass central lymph nodes. This anatomical structure could explain higher possibility of metastasis in tumors in upper pole.

Tumors no larger than $1 \mathrm{~cm}$ were more frequent in skip metastasis. In addition, Nie et al found that tumors $\leq 0.5$ $\mathrm{cm}$ tend to metastasize to the lateral neck instead of central compartment, while surgeries were proceeded in patients with a suspicious thyroid node $\leq 0.5 \mathrm{~cm}$ in the US report according to the ATA guideline. ${ }^{14,25}$ It seems that the arguments were against common sense, but this result agreed with what was concluded by Machens et al that skip metastasis was observed to be more recurrent in less aggressive forms of PTC. ${ }^{37}$

This study found that tumors without lymphovascular invasion were more likely to have skip metastasis. We considered only 2 studies were included and one study ${ }^{10}$ contributed mostly in fixed effect model (72.5\%). Thus, this result may not be that much convictive.

In particular, age $<45$ years was often referred as a risk factor for CLNM, but So et al found this was not significantly related to LLNM. ${ }^{20,44}$ In addition, age $<45$ years was also not associated with skip metastasis in our meta-analysis. According to $7^{\text {th }}$ AJCC TNM staging system, 45 years old was cut-off age and patients aging $<45$ could have lower risk factors for LNM. ${ }^{45}$ However, in view of the $8^{\text {th }}$ AJCC TNM staging system published in 2018, the cut-off age has been changed to 55 years old. Maybe there is a lower risk of skip metastasis for patients $<55$, but no research paper found about this aspect till now, so the age of 45 was applied in this analysis. Tumor multifocality is present in more than $80 \%$ PTC patients. ${ }^{46}$ However, tumor multifocality was unassociated with skip metastasis in our analysis. According to some previous reports, intraglandar spread was not associated with LLNM or skip metastasis. ${ }^{10,25,30}$ Tumor multifocality and intraglandar spread were considered as strong association before, but there is current debate about whether tumor multifocality in PTC represented the intraglandular spread of a single tumor or de novo occurrence of distinct tumors. They both were not significant of skip metastasis. Our analysis showed ETE was not significantly in line with skip metastasis. However, ETE has higher incidence of nodal disease in both central and lateral compartment according to the previous studies. A meta-analysis indicated that ETE was significantly connected with CLNM (pooled $\mathrm{OR}=2.10,95 \% \mathrm{CI}=1.81-2.43, \mathrm{P}<0.001) .{ }^{47}$ According to a meta-analysis in 2018, ETE presented a surprisingly high odds ratio for LLNM with or without CLNM (pooled OR = $3.22,95 \% \mathrm{CI}=2.21-4.70, \mathrm{P}<0.0001) .{ }^{48}$ We thought PTC with ETE was more likely to spread to central lymph nodes due to its spatially shorter distance. Therefore, when LNM happened, the central compartment had already been affected. In summary, skip metastasis was not dominant among patients with ETE in our analysis. 
Three hypotheses of skip metastasis were suggested by Lim et al in 2012: 1) Anatomical lymphatic channels existed that enables skip metastasis. 2) Normal anatomical lymphatic channels could be altered by neck surgeries. 3) There is false negative of limited or missed lymph node samples. ${ }^{10}$ In our analysis, included studies ruled out patients with neck operation history, so skip metastasis appeared to have developed through normal lymphatic systems bypassing the central lymph node compartment in our opinion. Further preclinical studies could be conducted for micro lymph structures in order to confirm these hypotheses. The significance of association between skip metastasis and PTC remained unclear. Previous studies have found that skip metastasis was more frequent in less aggressive PTC such as low-density LLNM and PTMC. ${ }^{10,11,37}$ Our included study found no significant difference of tumor-free survival of 450 PTC patients between 39 patients with skip metastasis and 411 patients without skip metastasis. ${ }^{26}$ Considered the similar form of skip metastasis in non-small-cell lung cancer and colorectal cancer, previous studies evaluated and compared the impact of skip metastases on tumor recurrence and survival, and then they obtained negative results. This indicated that skip metastasis may have a positive impact on prognosis. ${ }^{49,50}$ However, if this analogy was meaningful in PTC patients, it had to be studied afterward.

There are several limitations of our meta-analysis. Firstly, the number of included studies is limited, which may affect the result of our study, especially in the part of risk factor analysis. Secondly, all included studies were of retrospective design and our analysis was limited by their nature. Thirdly, most included studies were conducted in East-Asia except 3 articles which may cause some ethnic limitations.

\section{Conclusion}

In conclusion, the rate of skip metastasis was $12.02 \%$ in PTC patients with LNM. Therefore, the lateral compartment should be carefully examined especially for PTC patients who present primary tumors in the upper lobe, which have a primary tumor size $\leq 10 \mathrm{~mm}$, even though they have no obvious evidence of CNM. Whether skip metastasis is related to less aggressive PTC needs further preclinical and clinical studies.

\section{Acknowledgments}

Yuxuan Qiu and Yuan Fei are co-first authors of this paper.

\section{Disclosure}

The authors report no conflicts of interest in this work.

\section{References}

1. Morris LG, Sikora AG, Tosteson TD, Davies L. The increasing incidence of thyroid cancer: the influence of access to care. Thyroid. 2013;23(7):885-891.

2. Davies L, Welch HG. Current thyroid cancer trends in the United States. JAMA Otolaryngol Head Neck Surg. 2014;140(4):317-322.

3. La Vecchia C, Malvezzi M, Bosetti C, et al. Thyroid cancer mortality and incidence: a global overview. Int J Cancer. 2015;136(9):2187-2195.

4. Carling T, Udelsman R. Thyroid cancer. Annu Rev Med. 2014;65:125-137.

5. Robbins KT, Clayman G, Levine PA, et al. Neck dissection classification update: revisions proposed by the American Head and Neck Society and the American Academy of Otolaryngology-Head and Neck Surgery. Arch Otolaryngol Head Neck Surg. 2002;128 (7):751-758.

6. Wu MH, Shen WT, Gosnell J, Duh QY. Prognostic significance of extranodal extension of regional lymph node metastasis in papillary thyroid cancer. Head Neck. 2015;37(9):1336-1343.

7. Zeng RC, Zhang W, Gao EL, et al. Number of central lymph node metastasis for predicting lateral lymph node metastasis in papillary thyroid microcarcinoma. Head Neck. 2014;36(1):101-106.

8. Chung YS, Kim JY, Bae JS, et al. Lateral lymph node metastasis in papillary thyroid carcinoma: results of therapeutic lymph node dissection. Thyroid. 2009;19(3):241-246.

9. Paek SH, Lee YM, Min SY, Kim SW, Chung KW, Youn YK. Risk factors of hypoparathyroidism following total thyroidectomy for thyroid cancer. World J Surg. 2013;37(1):94-101.

10. Lim YC, Koo BS. Predictive factors of skip metastases to lateral neck compartment leaping central neck compartment in papillary thyroid carcinoma. Oral Oncol. 2012;48(3):262-265.

11. Park JH, Lee YS, Kim BW, Chang HS, Park CS. Skip lateral neck node metastases in papillary thyroid carcinoma. World J Surg. 2012;36(4):743-747.

12. Park JY, Koo BS. Individualized optimal surgical extent of the lateral neck in papillary thyroid cancer with lateral cervical metastasis. Eur Arch Otorhinolaryngol. 2014;271(6):1355-1360.

13. Goyal RM, Jonklaas J, Burman KD. Management of recurrent cervical papillary thyroid cancer. Endocrinol Metab Clin North Am. 2014;43(2):565-572.

14. Haugen BR, Alexander EK, Bible KC, et al. 2015 American Thyroid Association management guidelines for adult patients with thyroid nodules and differentiated thyroid cancer: the American Thyroid Association guidelines task force on thyroid nodules and differentiated thyroid cancer. Thyroid. 2016;26(1):1-133.

15. Leboulleux S, Girard E, Rose M, et al. Ultrasound criteria of malignancy for cervical lymph nodes in patients followed up for differentiated thyroid cancer. J Clin Endocrinol Metab. 2007;92(9):35903594.

16. Hartl DM, Leboulleux S, Al Ghuzlan A, et al. Optimization of staging of the neck with prophylactic central and lateral neck dissection for papillary thyroid carcinoma. Ann Surg. 2012;255(4):777783 .

17. Stulak JM, Grant CS, Farley DR, et al. Value of preoperative ultrasonography in the surgical management of initial and reoperative papillary thyroid cancer. Arch Surg. 2006;141(5):489-94; discussion 94-6.

18. Ali S, Tiwari RM, Snow GB. False-positive and false-negative neck nodes. Head Neck Surg. 1985;8(2):78-82.

19. Shin LK, Olcott EW, Jeffrey RB, Desser TS. Sonographic evaluation of cervical lymph nodes in papillary thyroid cancer. Ultrasound $Q$. 2013;29(1):25-32. 
20. So YK, Kim MJ, Kim S, Son YI. Lateral lymph node metastasis in papillary thyroid carcinoma: a systematic review and meta-analysis for prevalence, risk factors, and location. Int J Surg. 2018.;50:94103.

21. Stang A. Critical evaluation of the Newcastle-Ottawa scale for the assessment of the quality of nonrandomized studies in meta-analyses. Eur J Epidemiol. 2010;25(9):603-605.

22. Miller JJ. The inverse of the Freeman-Tukey double arcsine transformation. Am Stat. 1978;32(4):138.

23. Higgins JPT, Thompson SG, Deeks JJ, Altman DG. Measuring inconsistency in meta-analyses. Bmj. 2003;327(7414):557-560.

24. Jin WX, Jin YX, Ye DR, et al. Predictive factors of skip metastasis in papillary thyroid cancer. Med Sci Monit. 2018;24:2744.

25. Nie X, Tan Z, Ge M. Skip metastasis in papillary thyroid carcinoma is difficult to predict in clinical practice. BMC Cancer. 2017;17 (1):702.

26. Lei J, Zhong J, Jiang K, Li Z, Gong R, Zhu J. Skip lateral lymph node metastasis leaping over the central neck compartment in papillary thyroid carcinoma. Oncotarget. 2017;8(16):27022-27033.

27. Likhterov I, Reis LL, Urken ML. Central compartment management in patients with papillary thyroid cancer presenting with metastatic disease to the lateral neck: anatomic pathways of lymphatic spread. Head Neck. 2017;39(5):853.

28. Shi L, Song H, Zhu H, Li D, Zhang N. Pattern, predictors, and recurrence of cervical lymph node metastases in papillary thyroid cancer. Contemp Oncol. 2013;17(6):504-509.

29. Kliseska E, Makovac I. Skip metastases in papillary thyroid cancer. Coll Antropol. 2012;36 Suppl 2(36 Suppl 2):59-62.

30. Kim YS. Patterns and predictive factors of lateral lymph node metastasis in papillary thyroid microcarcinoma. Otolaryngol Head Neck Surg. 2012;147(1):15.

31. Koo BS, Choi EC, Park YH, Kim EH, Lim YC. Occult contralateral central lymph node metastases in papillary thyroid carcinoma with unilateral lymph node metastasis in the lateral neck. J Am Coll Surg. 2010;210(6):895.

32. Xiao GZ, Gao L. Central lymph node metastasis: is it a reliable indicator of lateral node involvement in papillary thyroid carcinoma? World J Surg. 2010;34(2):237.

33. Wada N, Masudo K, Nakayama H, et al. Clinical outcomes in older or younger patients with papillary thyroid carcinoma: impact of lymphadenopathy and patient age. Eur J Surg Oncol. 2008;34(2):202.

34. Roh JL, Kim JM, Park CI. Lateral cervical lymph node metastases from papillary thyroid carcinoma: pattern of nodal metastases and optimal strategy for neck dissection. Ann Surg Oncol. 2008;15 (4):1177-1182.

35. Roh JL, Park JY, Rha KS, Park CI. Is central neck dissection necessary for the treatment of lateral cervical nodal recurrence of papillary thyroid carcinoma? Head Neck. 2007;29(10):901-906.
36. Lee BJ, Wang SG, Lee JC, Son SM, Kim IJ, Kim YK. Level IIb lymph node metastasis in neck dissection for papillary thyroid carcinoma. Arch Otolaryngol Head Neck Surg. 2007;133(10):1028.

37. Machens A, Holzhausen HJ, Dralle H. Skip metastases in thyroid cancer leaping the central lymph node compartment. Arch Surg. 2004;139(1):43.

38. Lee YM, Sung TY, Kim WB, Chung KW, Yoon JH, Hong SJ. Risk factors for recurrence in patients with papillary thyroid carcinoma undergoing modified radical neck dissection. Br J Surg. 2016;103 (8):1020-1025.

39. Wada N, Duh QY, Sugino K, et al. Lymph node metastasis from 259 papillary thyroid microcarcinomas: frequency, pattern of occurrence and recurrence, and optimal strategy for neck dissection. Ann Surg. 2003;237(3):399-407.

40. Nixon IJ, Wang LY, Ganly I, et al. Outcomes for patients with papillary thyroid cancer who do not undergo prophylactic central neck dissection. Br J Surg. 2016;103(3):218-225.

41. Shaha AR. Complications of neck dissection for thyroid cancer. Ann Surg Oncol. 2008;15(2):397.

42. Grodski S, Cornford L, Sywak M, Sidhu S, Delbridge L. Routine level VI lymph node dissection for papillary thyroid cancer: surgical technique. ANZ J Surg. 2010;77(4):203-208.

43. Goropoulos A, Karamoshos KA, Ntitsias T, et al. Value of the cervical compartments in the surgical treatment of papillary thyroid carcinoma. World J Surg. 2004;28(12):1275.

44. Qu H, Sun GR, Liu Y, He QS. Clinical risk factors for central lymph node metastasis in papillary thyroid carcinoma: a systematic review and meta-analysis. Clin Endocrinol (Oxf). 2015;83(1):124-132.

45. Edge SB, Cancer Staging AJCC. Manual. JAMA. 2010;304 (15):1726-1727

46. Jovanovic L, Delahunt B, McIver B, Eberhardt NL, Grebe SK. Most multifocal papillary thyroid carcinomas acquire genetic and morphotype diversity through subclonal evolution following the intra-glandular spread of the initial neoplastic clone. J Pathol. 2008;215(2):145154.

47. Ma B, Wang Y, Yang S, Ji Q. Predictive factors for central lymph node metastasis in patients with $\mathrm{cN} 0$ papillary thyroid carcinoma: a systematic review and meta-analysis. Int J Surg. 2016;28:153-161.

48. So YK, Kim MJ, Kim S, Son Y. Lateral lymph node metastasis in papillary thyroid carcinoma: a systematic review and meta-analysis for prevalence, risk factors, and location. 2018;50:94-103. S1743919118300050.

49. Prenzel KL, Mönig SP, Sinning JM, et al. Role of skip metastasis to mediastinal lymph nodes in non-small cell lung cancer. J Surg Oncol. 2003;82(4):256-260.

50. Shiozawa M, Akaike M, Yamada R, et al. Clinicopathological features of skip metastasis in colorectal cancer. Hepatogastroenterology. 2007;54(73):81-84.

\section{Publish your work in this journal}

Cancer Management and Research is an international, peer-reviewed open access journal focusing on cancer research and the optimal use of preventative and integrated treatment interventions to achieve improved outcomes, enhanced survival and quality of life for the cancer patient.
The manuscript management system is completely online and includes a very quick and fair peer-review system, which is all easy to use. Visit http://www.dovepress.com/testimonials.php to read real quotes from published authors. 